\title{
Simulations of a Proof-of-Principle Experiment for Collinear Laser Spectroscopy within a Multi-Reflection Time-of-Flight Device
}

\author{
F. M. Maier · P. Fischer • H. Heylen • \\ V. Lagaki • S. Lechner • P. Plattner . \\ S. Sels · F. Wienholtz • W. Nörtershäuser • \\ L. Schweikhard · S. Malbrunot-Ettenauer
}

\begin{abstract}
For nearly four decades Collinear Laser Spectroscopy (CLS) has been employed to determine ground-state properties of short-lived radionuclides. To extend its reach to the most exotic radionuclides with very low production yields, the novel Multi Ion Reflection Apparatus for CLS (MIRACLS) is currently under development at ISOLDE/CERN. In this setup, 30-keV ion bunches will be trapped between two electrostatic mirrors of a multi-reflection time-of-flight (MR-ToF) device such that the laser beam will probe the ions during each revolution. Thus, the observation time will be extended and the experimental sensitivity will be increased significantly while maintaining the high resolution of conventional CLS. A proof-of-principle experiment is currently being performed to demonstrate the potential of CLS within a low-energy MR-ToF device. Its first experimental results benchmark the validity of ion-optical simulations from the CLS perspective, which will also be applied to MIRACLS' $30-\mathrm{keV}$ apparatus.
\end{abstract}

Keywords Multi-reflection time-of-flight device · Collinear Laser Spectroscopy • exotic radionuclides $\cdot$ MIRACLS

PACS 29.90.+r $\cdot 37.10 . T y \cdot 42.62 . F i$

F. Maier

JKU, Austria

E-mail: franziska.maier@hotmail.com

S. Malbrunot-Ettenauer · S. Sels

CERN, 1211 Geneva 23, Switzerland

Tel.: +41227674169

E-mail: stephan.ettenauer@cern.ch

P. Fischer · V. Lagaki · F. Wienholtz · L. Schweikhard

University Greifswald, Germany

P. Plattner

TU Innsbruck, Austria

S. Lechner

TU Wien, Austria

W. Nörthershäuser

TU Darmstadt, Germany 


\section{Introduction}

Due to its high accuracy and resolution, Collinear Laser Spectroscopy (CLS) is a powerful experimental technique to access nuclear ground-state properties such as nuclear spins, electromagnetic moments, and mean-square charge radii of shortlived radionuclides [1-3]. For CLS, a narrow-band laser beam is overlapped with a singly-charged ion or neutralized atom beam. When the wavelength of the laser matches the energy difference of the selected electronic transition, ions or atoms are excited. Resonance photons emitted by the excited ions or atoms can be detected in an optical detection region (ODR) by photomultiplier tubes (PMTs). By counting the number of photons as a function of the scanned laser frequency, the hyperfine structure of the optical transition is obtained. For high-resolution CLS, fast ion beams with a kinetic energy $E$ of tens of kiloelectronvolts are employed to minimize the Doppler broadening $\sigma_{f}$ according to $\sigma_{f} \propto \sigma_{E} / \sqrt{E}$ [4], where $\sigma_{E}$ is the energy spread of the ions. CLS provides model-independent information on nuclear ground-state properties. It is therefore an important experimental tool to benchmark modern nuclear theory and provides insight into nuclear structure phenomena. However, to experimentally probe the most exotic nuclides, which can only be produced with low production yields at today's radioactive ion beam facilities, new laser-spectroscopy techniques have to be envisioned to increase the sensitivity of conventional CLS.

This contribution will introduce the novel concept of the Multi Ion Reflection Apparatus for CLS of radionuclides (MIRACLS) currently being developed at ISOLDE/CERN. At MIRACLS, a bunched ion beam will be trapped between two electrostatic mirrors of a multi-reflection time-of-flight (MR-ToF) device [5-10], also called electrostatic ion beam trap [11-14]. The laser photons will interact with the ion bunch during each revolution. Therefore the observation time will be extended and the experimental sensitivity will be increased significantly compared to traditional CLS while maintaining its high resolution.

In the realm of rare-isotope science, MR-ToF instruments have recently gained remarkable attention, in particular for precision mass spectrometry or mass separation of short lived nuclides [6-10]. For high mass resolving powers, MR-ToF devices are operated with a focus in the time domain resulting in an increased energy spread of a few tens of electronvolt for kinetic beam energies $E$ of typically a few kiloelectronvolts [15]. However, for performing high-resolution CLS, $E$ is $\geq 30 \mathrm{keV}$ and the energy spread should be below $1 \mathrm{eV}$ in order to minimize the Doppler broadening and to obtain a spectral resolution which approaches the natural linewidth of the optical transitions. Moreover, CLS relies on a large ionlaser overlap with parallel ion and laser beam axes. Whereas in conventional CLS with a single ion passage through the ODR this can be readily achieved, an ion's trajectory in a MR-ToF device may vary from one revolution to the next (see below). Hence, MR-ToF operation modes have to be identified, which best fulfil the stringent CLS requirements to avoid broadening or distortions in the spectral line shape.

For this reason, simulations of the ions' trajectories in the envisioned $30-\mathrm{keV}$ MR-ToF apparatus are performed. These demonstrate that the requirements of CLS in a MR-ToF device can indeed be met. In order to validate these simulations and to demonstrate the functionality of the novel MIRACLS' approach a proofof-principle (PoP) experiment is currently carried out with a low-energy MR-ToF 
device [16-18], which has been modified for the purpose of CLS. Although the spectral resolution of CLS in the PoP experiment is due to the reduced beam energy of about $1.5 \mathrm{keV}$ about 4.5 times lower than the one of $30-\mathrm{keV}$ CLS, the comparison of the experimental data with the simulations will help to understand the observed line shapes under varying MR-ToF operation modes and will thus guide the design of MIRACLS' future MR-ToF apparatus. Moreover, experimental results of the PoP experiment will benchmark the validity of the simulation approach and provide confidence in its application to the $30-\mathrm{keV}$ MR-ToF device. Here, we will introduce MIRACLS' PoP experiment and its simulation from the CLS perspective.

\section{Experimental Setup for the Proof-of-Principle Experiment}

A general overview over the PoP setup is given in Fig. 1. An electron-impactionization ion source as described in [19] is modified to produce a continuous beam of stable ${ }^{24-26} \mathrm{Mg}^{+}$ions. Both transition $3 s^{2} S_{1 / 2} \rightarrow 3 p^{2} P_{1 / 2}$ and $3 s^{2} S_{1 / 2} \rightarrow$ $3 p^{2} P_{3 / 2}$ around $280 \mathrm{~nm}$, known as the D1 and D2 lines, form in the even-even ${ }^{24,26} \mathrm{Mg}^{+}$isotopes a closed two-level system, in which laser-excited ions decay back to the ionic ground-state. Hence, without optical pumping to other hyperfine states, these isotopes are ideal test cases for CLS in a MR-ToF device. While both transitions are probed during the PoP experiments, the present simulations are without loss in generality restricted to the D2 line.

After its formation, the continuous $250-\mathrm{eV}$ ion beam is injected into a compact, linear Paul trap [17] floated to $\sim 224 \mathrm{~V}$, where the ions are accumulated and cooled through collisions with helium buffer-gas atoms. The ions are ejected from the trap as well-defined ion bunches. A crown-shaped electrode accelerates these ion bunches towards a pulsed drift tube, which is switched from $-2080 \mathrm{~V}$ to ground

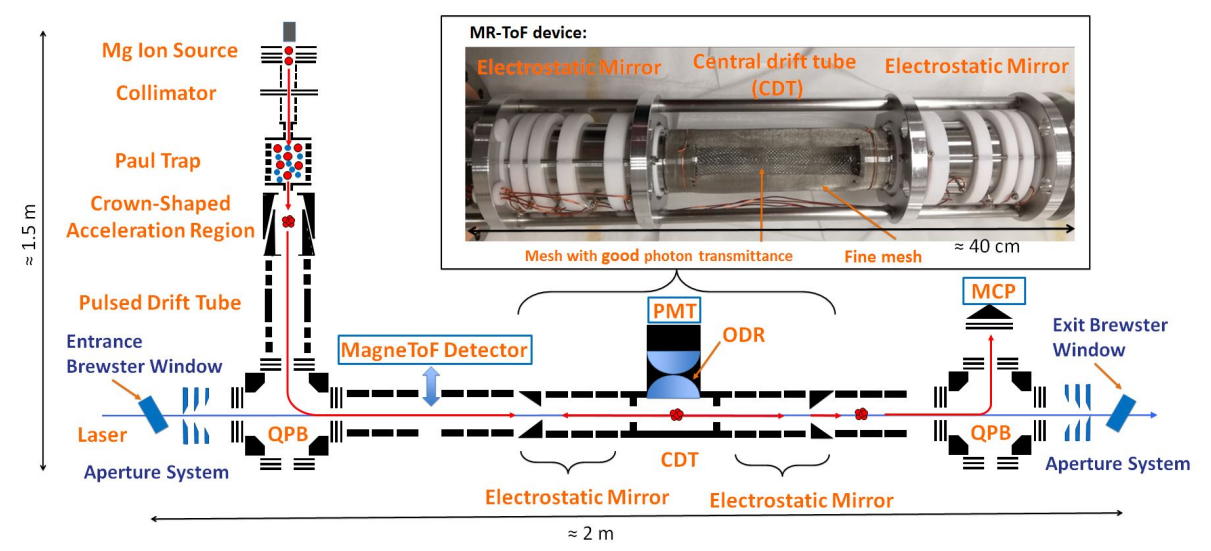

Fig. 1 Schematic drawing of the MIRACLS' proof-of-principle setup (not to scale). An ion source produces a continuous $\mathrm{Mg}^{+}$beam which is injected into a buffer-gas filled linear Paul trap for cooling, accumulation, and bunching. After acceleration, the ions are stored in the MRToF device. During each revolution the laser beam can interact with the ion bunch. Resonance photons are detected by a PMT installed above the central drift tube. Insert: Photo of the MR-ToF device. 
potential when the ions are in its center. Thus, the ions are lifted to a kinetic energy of $\sim 2.3 \mathrm{keV}$ with respect to the subsequent, grounded beam line. An electrostatic quadrupole bender (QPB) bends the ion beam onto the MR-ToF axis. A retractable MagneToF detector (14925, ETP Ion Detect, Ermington, Australia) is installed in front of the MR-ToF device for beam diagnostics.

The MR-ToF device consists of two opposing electrostatic ion mirrors positioned at either side of a central drift tube (CDT) (insert of Fig. 1). Each electrostatic mirror is composed of four cylindrical electrodes of which the one closest to the CDT is biased to a negative potential for transversal refocusing of the ions. The reflecting potential walls are realized by the three outer electrodes of each mirror. Given the sensitivity of the ions' trajectories to field distortions at their turn-around point, the outermost mirror electrode has a $12 \mathrm{~mm}$ diameter aperture at its outer end to shield the MR-ToF device from fringe fields of nearby electrodes.

The CDT consists of two electrodes and a connecting cylindrical, conductive mesh. At both ends of the CDT, the inner diameter is reduced to $14 \mathrm{~mm}$ compared to $40 \mathrm{~mm}$ inside the mirrors and the center of the CDT in order to shield the drift region from penetrating electric fields, providing a large, field-free region inside the CDT. Above the CDT, an optical lens system and a photomultiplier tube (modified from [20]) are installed to focus and detect, respectively, resonance photons emitted by laser-excited ions. Next to this ODR, the initial fine mesh is replaced by a mesh with higher transparency for an improved photon-detection efficiency.

For ion capture and ejection into and from the MR-ToF device, the technique of in-trap-lift switching is exploited [21]. Following its release from the trap, the ion bunch is bent by another QPB and detected by a multi-channel-plate (MCP) detector. Both QPBs facilitate the overlap between the ion and laser beam. The laser beam enters and exits the setup through quartz windows installed at the Brewster angles to minimize reflections.

\section{Simulations for the Proof-of-Principle Experiment}

\subsection{Identification of Suitable Mirror Potentials for CLS}

Simulations of the ions' trajectories in the MR-ToF device are carried out with SIMION (version 8.1.1.32)[22] with a focus on the requirements for CLS such as high ion-laser overlap and narrow spectral linewidth. Coulomb interactions between the different ions and between ions and image charges on the electrodes are not taken into account. To obtain a realistic distribution of the ions in the MR-ToF device, typically $200{ }^{24} \mathrm{Mg}^{+}$ions are created in the middle of the endcap hole of the Paul trap with a worst-case transversal emittance given by the geometry of the setup. The longitudinal emittance is varied separately (here $\sigma_{E}$ is in the order of $1 \mathrm{eV}$ ). After passing the acceleration and transfer section the ions are injected into the MR-ToF device with its mirror electrodes biased to a first trial combination of potentials known to perform well for mass measurements in this device. The ion distribution in the middle of the MR-ToF device (hence a field-free region) is recorded and taken as a starting ion distribution for a separate, dedicated simulation of the MR-ToF device itself (Fig. 2). Such a two-stage simulation approach is necessitated by limitations in computing power when considering the entire setup. By exclusively considering the MR-ToF device and taking advantage 


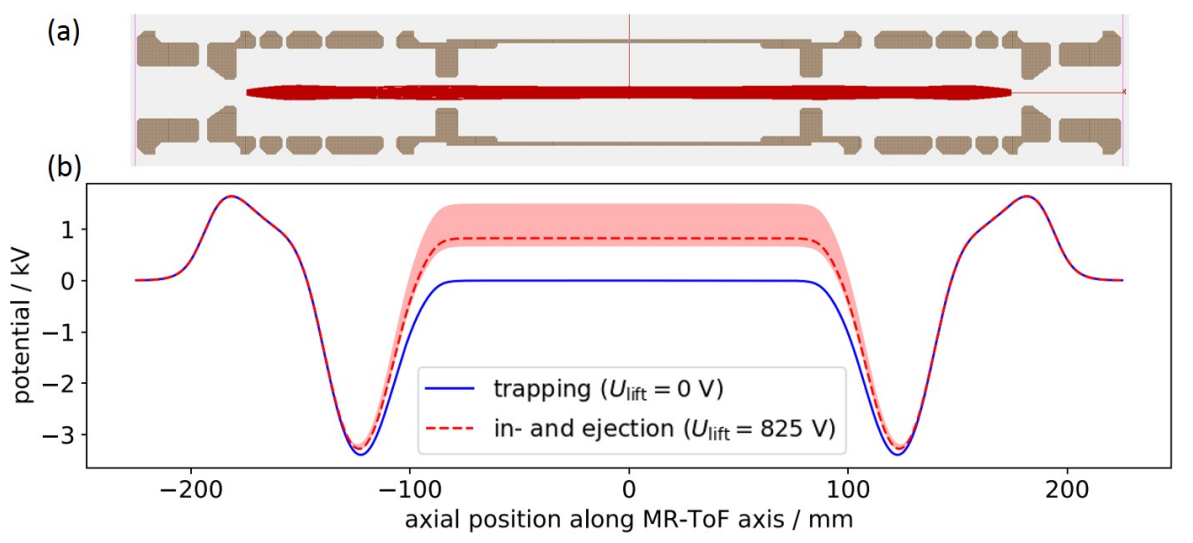

Fig. 2 MR-ToF device of MIRACLS' proof-of-principle experiment: (a) Cut view of its electrode structure together with the simulated ions' trajectories (in red) for 1000 ions performing 100 revolutions in the MR-ToF device. (b) Electric potential distribution along the central axis of the MR-ToF device for the chosen set of mirror potentials. The potential distribution used for trapping of the ions is shown in blue. All voltages of the central drift tube prior to the switch of the in-trap lift leading to close to $100 \%$ trapping efficiency are shown in red. The one that is chosen for the simulations is marked with a dashed line.

of its cylindrical symmetry in the second step of the simulation, the linear geometrical resolution can be increased by a factor of 10 compared to the first-step simulation of the entire setup. In order to identify suitable mirror potentials for CLS, a Monte Carlo approach is employed utilising the ion distribution generated as described above. In Fig. 3 the randomly chosen combinations of mirror potentials which lead to a 100\%-trapping efficiency for 20 revolutions in the MR-ToF device are shown. Each point corresponds to one potential combination that is characterized according to the mass resolving power, the spectral linewidth of the CLS spectra (Doppler width), and the ion-laser overlap, e.g. the fraction of ions with a transversal displacement $r$ smaller than the laser beam radius. All of these are evaluated when an ion passes the axial middle plane of the central drift tube in the direction of the laser-beam propagation during each revolution.

The Doppler width $\sigma_{f}$ is given by the standard deviation of the Doppler-shifted resonance frequencies $f$ in the lab frame,

$$
f=f_{0} \frac{\sqrt{1-\left(\frac{v}{c}\right)^{2}}}{1-\frac{v \cos \alpha}{c}},
$$

where $c$ is the speed of light, $v$ the velocity of the ions, and $\alpha$ is the angle between ion path and laser beam axis, assumed to be identical to the central axis of the MR-ToF device. The resonance frequency $f_{0}$ in the rest frame of the ${ }^{24} \mathrm{Mg}^{+}$ions is $(1,072,082,934.33 \pm 0.16) \mathrm{MHz}$ in the D2 line [23]. For this manuscript, we define the Doppler limit as the Doppler induced linewidth broadening exclusively due to the ions' velocity (hence energy) spread, that is in the limit of perfectly parallel ions' trajectories with respect to the laser beam. This is to distinguish it from the more general Doppler width which reflects the entire linewidth broadening due to 1 , so the spread in the angles between ions' trajectories and the laser beam as well as in energy. At the present beam energies of 1.3 to $1.7 \mathrm{keV}$, the spectral linewidth 


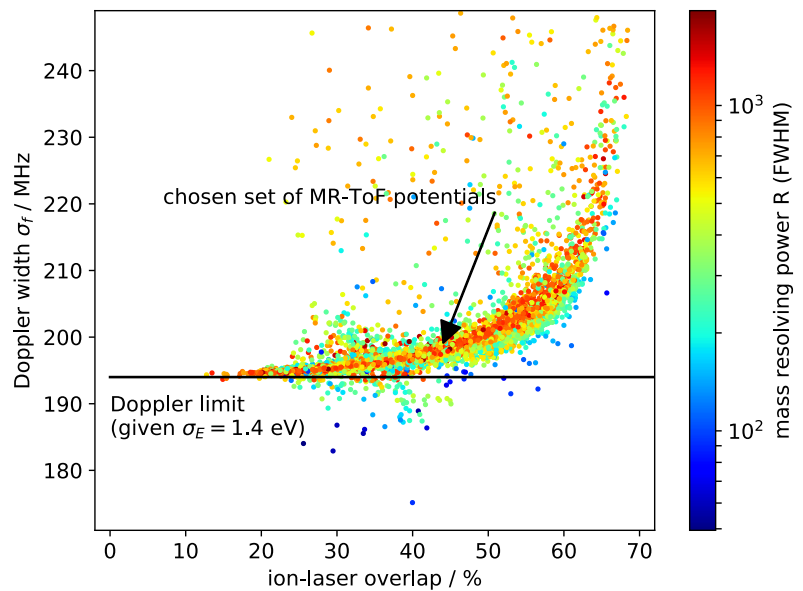

Fig. 3 Doppler width (standard deviation) and mass resolving power (FWHM) versus the ionlaser overlap (assumed laser diameter: $2 \mathrm{~mm}$ ) for different combinations of electric potentials of the MR-ToF mirrors. The selected set of potentials for the rest of this work is marked with an arrow. Note that potential combinations with a Doppler width of up to $300 \mathrm{MHz}$ were found that are not shown in this plot.

of conventional CLS spectra is essentially governed by the Doppler limit. However, for a MR-ToF device, a further investigation has to be carried out, since typically an ion passes the central drift tube at different angles $\alpha$ for different revolutions, which can lead to an additional broadening. As Fig. 3 shows, the Doppler width is increasing with better ion-laser overlap. This behaviour is interpreted as a consequence of the conserved transversal emittance $\Delta r \Delta \alpha$. For a given emittance of the beam coming from the Paul trap, an increase of $\Delta r$ implies that $\Delta \alpha$ is decreased and so is the Doppler width. However, the increase of $\Delta r$ also leads to a reduction of the overlap with the laser beam of limited fixed diameter. This observation emphasizes the importance of an excellent transversal emittance for highly sensitive and high-resolution CLS. Since the transversal emittance is governed by the beam preparation in the Paul trap [24], special attention will be given to this aspect of beam cooling and preparation in the future 30-keV MIRACLS apparatus. The calculated Doppler width for ions flying parallel to the MR-ToF axis (Doppler limit) is $\sigma_{f}=194 \mathrm{MHz}$ for the assumed energy spread of $\sigma_{E}=1.4 \mathrm{eV}$ and a kinetic energy of $\sim 1480 \mathrm{eV}$. In principle, this should be the smallest attainable Doppler width for any potential combination. However, for a few sets of potentials the ions penetrate further into the mirrors and the angles between MR-ToF axis and ion path tend to be correlated to the ions' velocities: Ions with large velocities have in these cases typically larger angles such that their resonance frequencies in the lab frame matches to the ones of ions with smaller velocities which tend to have smaller angles in their trajectories to the laser axis. Therefore, this correlation between $v$ and $\alpha$ leads, according to Eq. 1, to a compression in the resonance frequencies in the lab frame and the Doppler width can be smaller than the Doppler limit for these specific potential combinations (Fig. 3). 


\subsection{Simulations for a Chosen Set of Mirror Potentials}

In the following, a set of potentials with a good compromise in ion-laser overlap, Doppler width, and a reasonable mass resolving power is opted for. High mass resolving power is equivalent to short ion bunches which lead to high signal-tonoise ratios when gating the photon counting on the passage of the ion bunch. In figure 2(b) its resulting potential distribution along the MR-ToF axis is shown for trapping (in-trap lift potential $U_{\text {lift }}=0 \mathrm{~V}$ ) and for in- or ejection, where in-trap lift potentials between $665 \mathrm{~V}$ and $1500 \mathrm{~V}$ result in close to $100 \%$ trapping efficiency for 100 revolutions. For higher kinetic energies, the Doppler broadening is smaller and therefore in-trap lift potentials between $665 \mathrm{~V}$ and $870 \mathrm{~V}$ are preferred for the operation of the MR-ToF device in CLS mode. Within this potential range the difference in Doppler width is $\sim 35 \mathrm{MHz}$ and in ion-laser overlap $\sim 12 \%$. Thus, an in-trap lift potential of $825 \mathrm{~V}$ is selected for the simulations discussed below.

Employing the selected set of MR-ToF mirror potentials and the chosen bias voltage of the in-trap lift, the ion beam injection from the Paul trap into the MRToF device is repeated. This confirms that the ion distribution obtained in the middle of the MR-ToF device is very similar to the ion ensemble utilised in the previous Monte-Carlo based probing of MR-ToF mirror potentials.

While all CLS simulations discussed so far assumed ions to be in the middle transversal plane of the MR-ToF device, photons emitted by ions within $\sim 45 \mathrm{~mm}$ on both sides of the center can also be detected efficiently [20]. However, only in a completely field-free region, the ion velocity is independent of its position inside the ODR. In practice, remaining potential gradients result in slightly different velocities and hence different laser frequencies in the ions' rest frame. As shown in Fig. 4, in a region of $92 \mathrm{~mm}$, the change in electric potential is less than $0.04 \mathrm{~V}$ corresponding to a change of the Doppler-shifted frequency of less than $5 \mathrm{MHz}$ for an ion kinetic energy of $1500 \mathrm{eV}$. Given the lifetime of around $3.8 \mathrm{~ns}$ [25] of the excited states of the D1 and D2 transitions for the $\mathrm{Mg}^{+}$ions and the kinetic energy in the MR-ToF device of 1.3 to $1.7 \mathrm{keV}$, an excited ion will typically move less than $0.5 \mathrm{~mm}$ until it decays back to the ground-state. Hence, ions potentially excited outside the ODR at different velocities (and therefore other resonance frequencies in the lab frame) will not be detected. In order to implement more

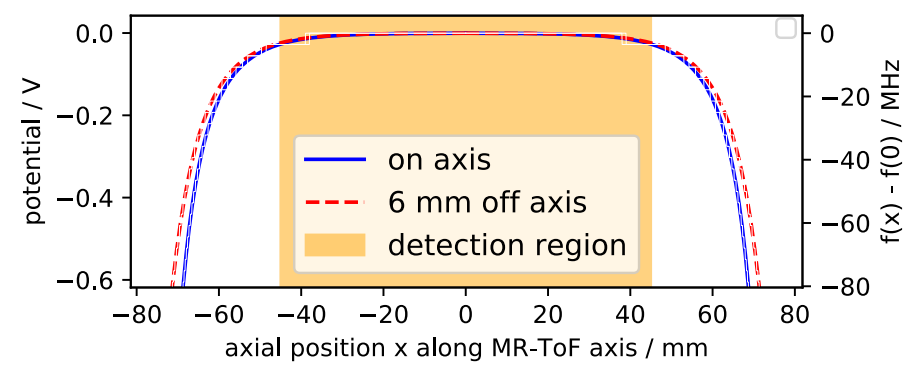

Fig. 4 Electric potential and the change of the Doppler-shifted frequency $f(x)-f(0)$ for a kinetic energy of $1479 \mathrm{eV}$ once for a ${ }^{24} \mathrm{Mg}^{+}$ion flying along the central axis of the MR-ToF device and once on a parallel line shifted $6 \mathrm{~mm}$ off axis. The length of the ODR is marked in orange. The central drift tube itself is reaching from -106 to $106 \mathrm{~mm}$. 

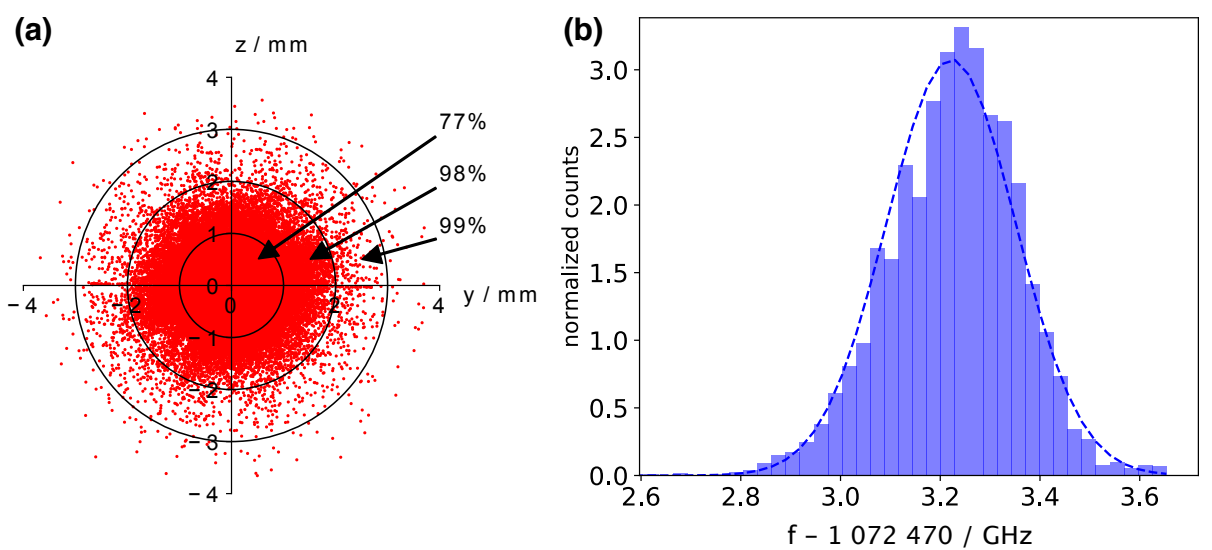

Fig. 5 CLS-relevant results when ions are stored in the MR-ToF device for 100 revolutions: (a) Transversal distances of the ions from the central axis of the MR-ToF device. A circle with a diameter of $2 \mathrm{~mm}$, i.e. a typically laser beam size, spans over $77 \%$ of the ions. (b) Histogram of the Doppler-shifted frequency for CLS only considering the laser-excited ions. A Gaussian line shape with centroid and width extracted from the data shows a slight shape asymmetry. See text for details.

realistic conditions for the transversal and longitudinal emittance, simulations of the ion preparation inside the helium-filled Paul trap are performed. 1000 ions are randomly distributed around the trapping position of the Paul trap and thermalized through collisions with helium buffer-gas atoms at a pressure of $10^{-3}$ mbar. The ion-buffer-gas interactions are based on a hard-sphere collision model implemented in SIMION. After $2 \mathrm{~ms}$, the ions are ejected from the Paul trap and their distribution is recorded in the middle plane of the acceleration zone and taken as a starting ion distribution for the previously discussed two-stage simulation. Some ions undergo collisions with buffer-gas atoms during the extraction from the Paul trap, which results in different kinetic energies and different angles and hence they are lost in the MR-ToF device within the first three revolutions. Hence, the trapping efficiency is $\sim 97 \%$. Once again, the ion distribution is recorded for each revolution when the ions pass the transversal middle plane in the direction of the laser beam propagation.

From this data, the transversal distances of the ions in $y$ and $z$ to the central axis of the MR-ToF device ( $x$-axis) are shown in Fig. 5(a) for 100 revolutions. A laser beam with a diameter of $2 \mathrm{~mm}$ overlaps with $77 \%$ of the ions. Such a laser beam diameter is typically chosen in the experiment to keep the laser-induced stray-light background at a reasonable level. Exemplary for all simulated ions, sample ions are tracked for 2000 revolutions as they move in regular patterns through the MR-ToF device's transversal middle plane (Fig. 6(a)) with oscillating angles of flight trajectories to the MR-ToF axis (Fig. 6(b)). For the simulated 1000 ions and a laser beam diameter of $2 \mathrm{~mm}$, a histogram of the Doppler-shifted frequencies of the D2 transition in ${ }^{24} \mathrm{Mg}^{+}$summed over 100 revolutions is shown in Fig. 5(b). The Doppler width due to the energy spread and varying angles between ions' trajectories and laser beam is $160 \mathrm{MHz}$ compared to the Doppler limit of $156 \mathrm{MHz}$ for the energy spread of $1.2 \mathrm{eV}$ of all laser-excited ions. A non-vanishing angle between ions' trajectories and laser beam axis will shift the laser frequency 
observed in the ion's rest frame according to Eq. 1 in one direction, i.e. to lower values for collinear laser spectroscopy (and to higher values for the anti-collinear case which is not considered here). This results in an asymmetric line shape. However, as seen in Fig. 5(b), this asymmetry is not pronounced since the angles between ions' trajectories and laser axis are small (average value of 0.4 degree, standard deviation of 0.4 degree) leading to shifts in mean values of typically only $12 \mathrm{MHz}$. The evolution of the ion-laser overlap and the Doppler width with increasing revolution number can be seen in Fig. 7(a) and (b), respectively. It typically takes some tens of revolutions until the ions are accommodated to stable ions' trajectories in the MR-ToF device. After around 40 revolutions, however, the ion distribution at the middle plane of the MR-ToF device remains largely identical for consecutive revolutions. This 'acclimatization' process over the first few tens of revolutions is readily apparent in the ion-laser overlap (Fig. 7(a)). The Doppler width for ions excited by a laser beam of $2 \mathrm{~mm}$ diameter is fluctuating between 130 and $200 \mathrm{MHz}$ (Fig. 7(b)). For a larger laser beam spot covering all ions, the Doppler width is rather stable around $228 \mathrm{MHz}$, when the first three revolutions are neglected. The initial drop from 270 to $228 \mathrm{MHz}$ is due to the loss of unsuitable ions within the first revolutions.

In light of the limitations of the hard-sphere collision model [26] and our incomplete knowledge about the helium pressure in the Paul trap, these results are compared in Tab. 1 to the initially introduced simulation scenario based on a worst-case transversal emittance. The results differ significantly only for the ion-laser overlap reflecting the large difference in transversal emittance. However, determining the experimental ion-laser overlap is not straightforward. The spectral linewidth is dominated by the ions' energy spread in both cases, providing confidence that line shape distortions due to the MR-ToF characteristics are well under control in properly chosen settings.
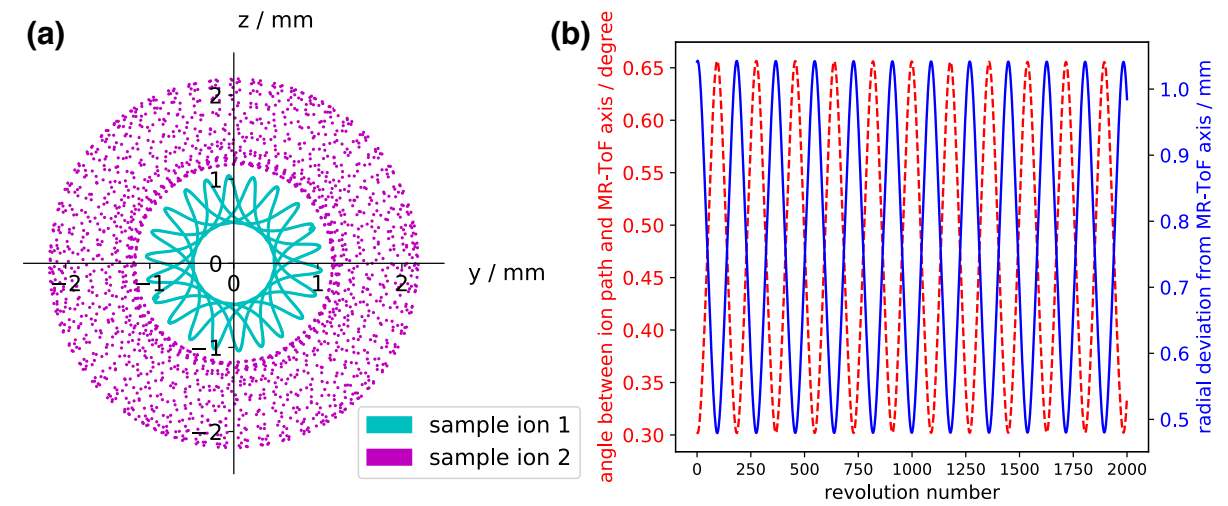

Fig. 6 (a) Transversal distances from the MR-ToF axis for two ions with different starting parameters (different angle to MR-ToF axis and different transversal distance to MR-ToF axis) for 2000 revolutions. (b) Transversal distance from the MR-ToF axis (blue) and angle between ion path and MR-ToF axis (red) versus revolution number for the first ion. The amplitude, phase, and frequency depend on the ion's starting parameters as well as on the potential combination of the mirror electrodes. 


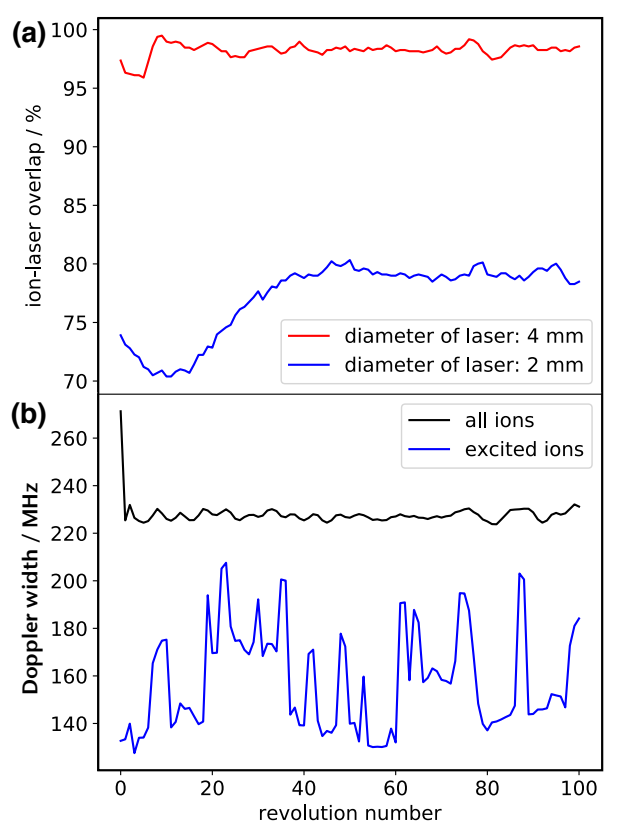

Fig. 7 Ion-laser overlap (a) and Doppler width (b) versus revolution number. See text for details.

Table 1 Summary of the simulation of the PoP experiment including the ions' thermalization in the Paul trap (right column, complete scenario) and the scenario with the worst-case transversal emittance (left column, worst-case scenario). For the latter, an energy spread of $1.5 \mathrm{eV}$ was assumed. In the MR-ToF device, the kinetic energy is $\sim 1480 \mathrm{eV}$ and a laser beam size of $2 \mathrm{~mm}$ diameter is assumed. Doppler widths, energy and angular spread are given in one standard deviation. Both simulations are performed with 1000 ions and 100 revolutions.

\begin{tabular}{|c|c|c|}
\hline & \multicolumn{2}{|c|}{ worst-case scenario | complete scenario } \\
\hline transversal rms emittance & $5.3 \pi \mathrm{mm} \mathrm{mrad}$ & $0.9 \pi \mathrm{mm} \mathrm{mrad}$ \\
\hline trapping efficiency & $100 \%$ & $97.6 \%$ \\
\hline erage angle $\alpha$ between ion path and laser axis & 0.8 degree & 0.4 degree \\
\hline spread of $\alpha$ & 0.5 degree & 0.4 degree \\
\hline ion-laser overlap & $47 \%$ & $77 \%$ \\
\hline energy spread (all ions) & $1.5 \mathrm{eV}$ & $1.7 \mathrm{eV}$ \\
\hline energy spread (excited ions) & $1.4 \mathrm{eV}$ & $1.2 \mathrm{eV}$ \\
\hline spectral linewidth (all ions): & & \\
\hline Doppler limit & $194 \mathrm{MHz}$ & $224 \mathrm{MHz}$ \\
\hline Doppler width & $201 \mathrm{MHz}$ & $228 \mathrm{MHz}$ \\
\hline spectral linewidth (excited ions): & & \\
\hline Doppler limit & $188 \mathrm{MHz}$ & $156 \mathrm{MHz}$ \\
\hline Doppler width & $196 \mathrm{MHz}$ & $160 \mathrm{MHz}$ \\
\hline
\end{tabular}

\section{Comparison with First Experimental Data}

A first benchmark of the simulation is found in the time-of-flight (ToF) distribution of the ions measured by the MagneToF detector installed between the QPB and the MR-ToF device. A FWHM in the ToF peak of 199(12)ns is measured. Its 


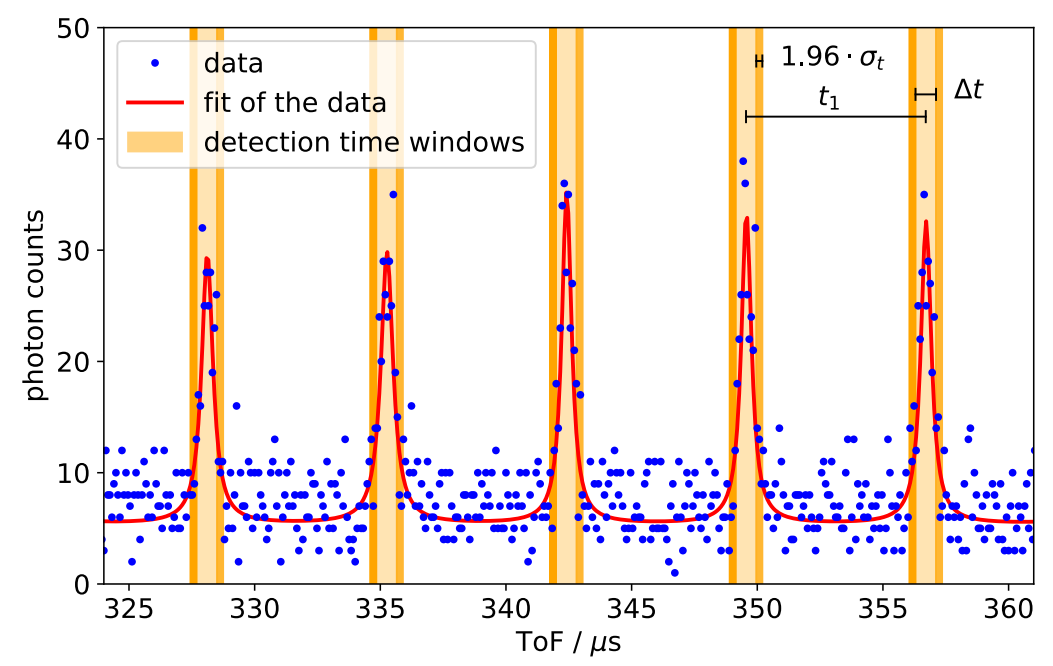

Fig. 8 Detected photon counts per time bin of 80 ns versus the ToF of the ions since extraction from the Paul trap for 100 ion shots. The photon peaks result from resonance photons associated with the passage of the ${ }^{24} \mathrm{Mg}^{+}$ion bunch through the ODR. The photon peaks were fitted with a Gaussian line shape to guide the eye. The exact temporal peak shape is still under evaluation. The simulated time duration of one revolution $t_{1}$ is indicated together with the time $\Delta t$ a single ion needs to pass the ODR (light orange). The ions' ToF distribution causes an additional extension of the detection time windows, of which the approximative size is indicated by dark orange bands on both sides of the photon peaks. The duration of the combined orange bands corresponds to a $95 \%$ width of the ions' simulated ToF peak, given by $3.92 \sigma_{t} \approx 485 \mathrm{~ns}$ for the shown revolution numbers of $45-49$.

agreement with the value of $194 \mathrm{~ns}$ predicted by the simulation provides confidence in the Paul-trap simulation. Next, the MR-ToF mirror potentials identified as suitable for CLS are tested experimentally. Ions are stored successfully in the MRToF device for more than 3,000 revolutions, corresponding to a trapping time of $\sim 22 \mathrm{~ms}$. The ions cannot be efficiently captured if the potential of the central drift tube, prior to the switching to ground, is less than $\sim 650 \mathrm{~V}$, which is in good agreement with the simulated value of $665 \mathrm{~V}$.

Moreover, first CLS measurements are performed within the low-energy PoP MR-ToF device (with an in-trap lift potential of $795 \mathrm{~V}$ ). Fig. 8 shows the number of detected photons as a function of the ToF of the ions since their extraction from the Paul trap for a laser frequency matching the resonance frequency. The passage of ${ }^{24} \mathrm{Mg}^{+}$ions through the ODR is apparent in the photon counts. According to the simulations, the ions need a time of $\Delta t \sim 820 \mathrm{~ns}$ from one end of the detection region to the other. The simulated time spread of the ion ensemble for the shown revolution numbers 45 to 49 is $\sigma_{t} \sim 125 \mathrm{~ns}$. This leads to an increase of the duration of the fluorescence signals (see Fig. 8), which is in good agreement with the experimental ToF width of the recorded resonance-photon counts. The simulated time for one revolution $t_{1}$ for an in-trap lift potential of $795 \mathrm{~V}$ is $7.152(2) \mu \mathrm{s}$, which is in line with the experimental distance between the ToF peaks of 7.15(5) $\mu \mathrm{s}$. 


\section{Summary and Outlook}

Simulations of the ions' trajectories in MIRACLS' low-energy PoP MR-ToF device have been performed to investigate the laser-spectroscopic line broadening and the ion-laser overlap. The first experimental results show a good agreement with the simulations. Both simulations and first experimental results demonstrate that CLS with a MR-ToF device can enhance the sensitivity of conventional CLS (by a factor depending on the case-to-case details such as the nuclides' lifetime). With sets of mirror potentials optimized for the requirements of CLS, the shape of the simulated spectral line is neither broadened nor significantly distorted by the combination of CLS and MR-ToF operation. The first experimental results are in line with the simulations and benchmark the validity of the simulation approach which is currently applied to MIRACLS' future 30-keV MR-ToF device. This apparatus will operate at ion beam energies of $30 \mathrm{keV}$ to minimize the Doppler broadening such that the spectral linewidth is comparable to the natural linewidth. In addition, this will be the first MR-ToF setup operating with such high electric potentials. We are currently also studying the advantages and new opportunities beyond collinear laser spectroscopy, in particular with respect to precision mass spectrometry and mass separation of rare isotopes.

Acknowledgements The research leading to these results has received funding from the European Research Council (ERC) under the European Unions Horizon 2020 research and innovation programme under grant agreement No 679038. P.F. and L.S. acknowledges support by the German Ministry for Education and Research (BMBF, 05P15HGCIA). We would like to express our gratitude to K. Blaum for many fruitful discussions and his continuous support to the project. We thank M. Bissell, M. Borge, J. Dilling, R. Garcia, M. Kowalska, R. Neugart, G. Neyens, M. Rosenbusch, R. Sanchez, and R. Wolf, for their help and advice, especially during the beginning of the project. We are grateful to Z. Andjelkovic, T. Murbc̈k, and S. Schmidt for sharing their experience on a compact $\mathrm{Mg}$ ion source as well as to S. Sailer and L. Bartels for their earlier experimental contributions.

\section{References}

1. K. Blaum, J. Dilling, W. Nörtershäuser, Physica Scripta 2013(T152), 014017 (2013)

2. P. Campbell, I. Moore, M. Pearson, Progress in Particle and Nuclear Physics 86, 127 (2016)

3. R. Neugart et al, Journal of Physics G: Nuclear and Particle Physics 44(6), 064002 (2017)

4. S. Kaufman, Optics Communications 17(3), 309 (1976)

5. H. Wollnik, M. Przewloka, International Journal of Mass Spectrometry and Ion Processes 96(3), 267 (1990)

6. W. R. Plass et al, Nuclear Instruments and Methods in Physics Research Section B: Beam Interactions with Materials and Atoms 266(19), 4560 (2008)

7. A. Piechaczek et al, Nuclear Instruments and Methods in Physics Research Section B: Beam Interactions with Materials and Atoms 266(19), 4510 (2008)

8. P. Schury et al, Eur. Phys. J A 42,343 (2009)

9. R.N. Wolf et al, Nuclear Instruments and Methods in Physics Research Section A: Accelerators, Spectrometers, Detectors and Associated Equipment 686, 82 (2012)

10. F. Wienholtz et al, Nature 498, 346 (2013)

11. D. Zajfman et al, Phys. Rev. A 55, R1577 (1997)

12. W.H. Benner, Anal. Chem. 69(20), 4162 (1997)

13. J. D. Alexander et al, Journal of Physics B: Atomic, Molecular and Optical Physics 42(15), 154027 (2009)

14. M. Lange et al, Review of Scientic Instruments 81(5), 055105 (2010)

15. R.N. Wolf et al, International Journal of Mass Spectrometry 349-350, 123 (2013)

16. M. Rosenbusch et al, AIP Conference Proceedings 1521(1), 53 (2013) 
17. M. Rosenbusch et al, AIP Conference Proceedings 1668(1), 050001 (2015)

18. L. Schweikhard et al, Proceedings of Science PoS (X LASNPA) 011 (2014)

19. T. Murböck et al, Review of Scientic Instruments 87(4), 043302 (2016)

20. K. Kreim et al, Physics Letters B 731, 97 (2014)

K. Kreim, Ph.D. thesis, Ruprecht-Karls-Universitt Heidelberg, (2013)

21. R. N. Wolf et al, International Journal of Mass Spectrometry 313, 8 (2012)

22. D. Manura and D. Dahl. Simion (r) 8.1 user manual (2013)

23. V. Batteiger et al, Phys. Rev. A 80, 022503 (2009)

24. R. B. Moore et al, Physica Scripta 1995(T59), 93 (1995)

25. W. Ansbacher, Y. Li, E. Pinnington, Physics Letters A 139(3), 165 (1989)

26. S. Schwarz, Nuclear Instruments and Methods in Physics Research Section A: Accelerators, Spectrometers, Detectors and Associated Equipment 566(2), 233 (2006) 